\title{
Fatigue Tests and Fracture Behavior Analysis of Porous Implant Materials Fabricated by 3D Metal Printing Technology
}

\author{
Ming-Hsien $\mathrm{Hu},{ }^{1}$ Chun-Ming Chang, ${ }^{2}$ Tan-Chih Chang, ${ }^{3}$ \\ Yao-Tsung Yang, ${ }^{4}$ Chia-Hui Chien, ${ }^{5}$ Wan Ao, ${ }^{4}$ and Feng-Min Lai ${ }^{4 *}$ \\ ${ }^{1}$ Department of Orthopedic Surgery, Show Chwan Memorial Hospital, \\ No. 542, Sec. 1 Chung-Shan Rd., Changhua, Taiwan (R.O.C.) \\ ${ }^{2}$ Taiwan Instrument Research Institute, 20 R\&D Rd VI. Hsinchu 30076, Taiwan (R.O.C.) \\ ${ }^{3}$ Department of Mechanical and Automation Engineering, Da-Yeh University, \\ No. 168, University Rd., Dacun, Changhua City 515, Taiwan (R.O.C.) \\ ${ }^{4}$ Bachelor Program for Design and Materials for Medical Equipment and Devices, Da-Yeh University, \\ No. 168, University Rd., Dacun, Changhua, Taiwan (R.O.C.) \\ ${ }^{5}$ Department of Dentistry, Chi-Mei Hospital, \\ Liouying No.201, Taikang Vil., Liuying Dist., Tainan City 71004, Taiwan (R.O.C.)
}

(Received January 4, 2021; accepted May 27, 2021)

Keywords: 3D printing of porous materials, mechanical properties, fatigue testing, heat treatment, manufacturing process

3D-printed Ti-6Al-4V materials can be used for implantation upon the confirmation of medical device specifications. Moreover, Young's coefficient of porous Ti-6Al-4V is close to that of the human bone, and it can allow the growth of bone cells through holes to fuse with implant materials. In this study, porous Ti-6Al-4V samples were fabricated by 3D printing. The fabricated porous Ti-6Al-4V samples had four rows of holes in the front and one or two rows of holes in the side. The mechanical properties and fatigue lifetimes of the porous Ti-6Al-4V samples were analyzed, where the samples were prepared by selective laser melting (SLM) 3D printing followed by post-heat treatment at a temperature of 500,700 , or $925{ }^{\circ} \mathrm{C}$ in a vertical tube furnace for $2 \mathrm{~h}$ with a heating rate of $10{ }^{\circ} \mathrm{C} / \mathrm{min}$. A tensile testing machine was used to measure Young's modulus and tensile strength of the porous Ti-6Al-4V alloy samples. Upon microstructural observation by SEM, the as-fabricated 3D-printed sample showed a fine needlelike martensite structure, and the 500 - and $700-^{\circ} \mathrm{C}$-treated samples showed a needle-like $\beta$-structure. Additionally, the $925-^{\circ} \mathrm{C}$-treated sample showed a coarse $\beta$-structure. The porous Ti-6Al-4V sample subjected to post-heat treatment at $500{ }^{\circ} \mathrm{C}$ had the lowest Young's modulus and the highest tensile strength, where the strengths used in the fatigue testing were $0.1,0.2$, and 0.6 times the yield strength. The fatigue lifetimes of the porous Ti-6Al-4V samples with one and two rows of holes in the lateral side were 128941 and 22721 cycles at 0.2 times the yield strength, respectively.

*Corresponding author: e-mail: fengmin@mail.dyu.edu.tw https://doi.org/10.18494/SAM.2021.3291 


\section{Introduction}

Ti-6Al-4V is an $\alpha+\beta$ titanium alloy used in medical implants. This alloy has many advantages such as a light weight, high temperature resistance, corrosion resistance, low thermal expansion coefficient, nonmagneticity, and nontoxicity. ${ }^{(1)}$ However, the mechanical properties of pure titanium alloy are much stronger than those of human bone. Stress concentration can thus easily occur when titanium alloy implants are used, leading to the damage and breakage of bones. ${ }^{(2)}$ Therefore, in this study, to fabricate Ti-6Al-4V alloys with Young's coefficient close to that of human bone, porous Ti-6A1-4V samples with various hole arrangements were prepared by the selective laser melting (SLM) 3D printing technique.

Note that, owing to the attractive properties of Ti-6Al-4V alloys, they can also be used to fabricate various sensor devices, such as optical fiber sensors, biosensors, piezoelectric force sensors, and acoustic emission sensors. Regardless of whether Ti-6Al-4V alloys are used for biomedical or sensor applications, suitable mechanical properties are important to extend their lifetime. Thus, in this study, we have focused on the mechanical properties of SLM-prepared Ti-6Al-4V alloys.

In contrast to the computer numerical control $(\mathrm{CNC})$ subtractive method (a subtractive process), the SLM 3D printing technique is an additive process, which can reduce the amount of waste of powder material. The mechanical strengths of SLM-prepared samples are almost the same as those obtained by casting. In this study, the number of rows of holes in the SLM-prepared samples and the temperature of the post-heat treatment were both modulated, and the subsequent variations in the morphology, mechanical strength, and fatigue lifetime of the samples were analyzed..$^{(3,4)}$ The purpose of this study is described as follows.

(1) Porous titanium alloy samples were prepared by 3D printing, and the mechanical properties of samples with various hole designs (horizontal, diameter, and number of side rows) were evaluated.

(2) Post-heat treatments were performed on the 3D-printed samples, and the effect of the heat treatment temperature on the mechanical properties was analyzed. ${ }^{(5)}$

(3) The variations in the yield strength, Young's coefficient, elongation, and fatigue lifetime of the samples with various hole designs were analyzed by performing tensile and fatigue tests.

(4) To clarify the relationship among the crystal structure, mechanical properties, fatigue lifetime, and cross-sectional morphology of the 3D-printed samples, their microstructures were observed by optical microscopy (OM) and scanning electron microscopy (SEM) ${ }^{(6,7)}$

\section{Experimental Method}

In this study, the 3D-printed porous Ti-6Al-4V samples were fabricated by SLM (Taiwan Instrument Research Institute, Taiwan). The effect of the post-heat treatment on the microstructural and mechanical properties of these Ti-6Al-4V samples was investigated in detail. The experimental process followed to obtain both the optimum porous structure (hole design) and the heat treatment conditions of the 3D-printed Ti-6Al-4V samples is shown in Fig. 1. 


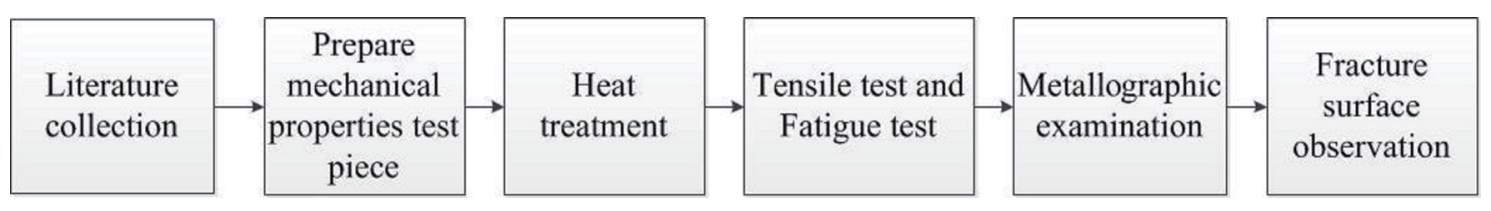

Fig. 1. Experimental process.

\subsection{Preparation of samples}

The porous Ti-6Al-4V samples were fabricated by following the ASTM E8/E8M printing specifications. As shown in Fig. 2, the SLM-prepared porous Ti-6Al-4V samples were all printed in the vertical direction. The porous Ti-6Al-4V samples had four rows of holes in the front side and one or two rows of holes in the lateral side (as shown in Fig. 3).

\subsection{Heat treatment}

To improve the mechanical properties of the 3D-printed porous Ti-6Al-4V samples, post-heat treatments were performed at annealing temperatures of 500,700 , and $925^{\circ} \mathrm{C}$ for 4,2 , and $1 \mathrm{~h}$, respectively. The heating rate was fixed at $10{ }^{\circ} \mathrm{C} / \mathrm{min}$ during the post-heat treatment.

\subsection{Mechanical properties test}

We found that the measured mechanical properties of the Ti-6Al-4V samples were markedly affected by their rough surface. ${ }^{(6,8)}$ Therfore, before performing tensile and fatigue tests, the four planes at the necks of the porous Ti-6Al-4V samples were completely ground, ${ }^{(9)}$ as shown in Fig. 4. In the tensile tests, the tensile direction was perpendicular to the width direction of the Ti-6Al-4V sample and the tensile rate was kept at $1 \mathrm{~min} / \mathrm{mm}$. The yield strengths of these porous Ti-6Al-4V samples were obtained from the tensile test results. Strengths of $0.1,0.2$, and 0.6 times the yield strength of the samples were used in the fatigue tests, which were performed using the setup shown in Fig. 5. In the fatigue tests, a working frequency of $3 \mathrm{~Hz}$ was employed and the stress ratio $\left(R=\sigma_{\min } / \sigma_{\max }\right)$ was -1 .

\section{Results and Discussion}

\subsection{Effect of heat treatment on tensile and fatigue properties}

Table 1 shows the results of tensile tests on the Ti-6Al-4V samples with one and two rows of holes in the lateral side. The tensile strength of the $500-^{\circ} \mathrm{C}$-treated sample was higher than that of the 700 - and $925-{ }^{\circ} \mathrm{C}$-treated samples. In addition, fatigue tests were performed on the two porous Ti-6Al-4V samples with 0.15 and 0.3 times the yield strength. It was found that the fatigue lifetime of the porous Ti-6Al-4V sample with one row of holes in the lateral side was 150000 cycles when 0.15 times the yield strength was used, while that of the sample with two 


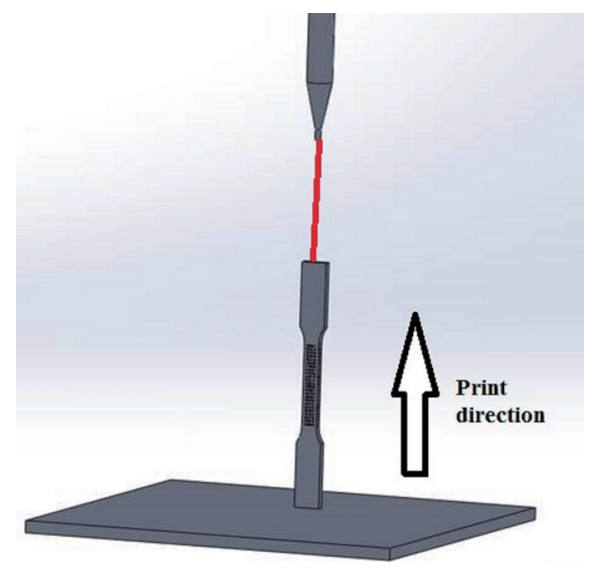

Fig. 2. (Color online) Printing direction of samples.

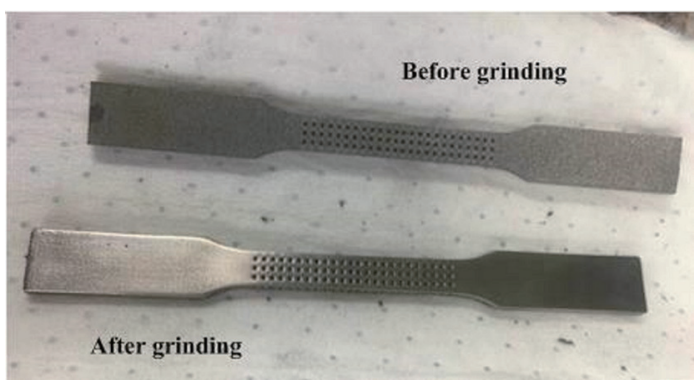

Fig. 4. (Color online) 3D-printed Ti-6Al-4V samples before and after grinding.

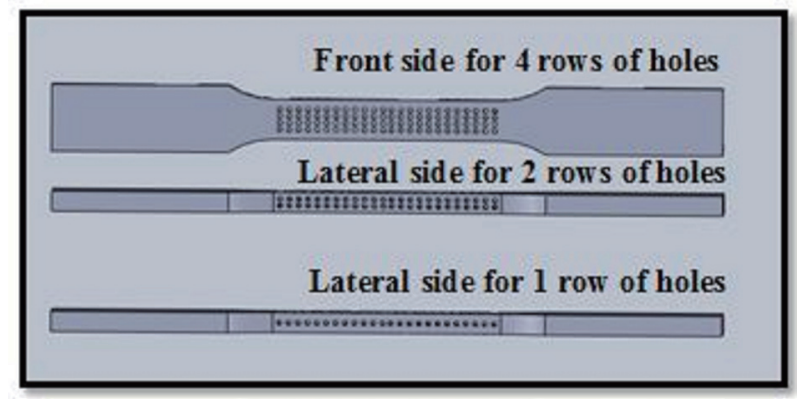

Fig. 3. Samples with different numbers of rows of holes.

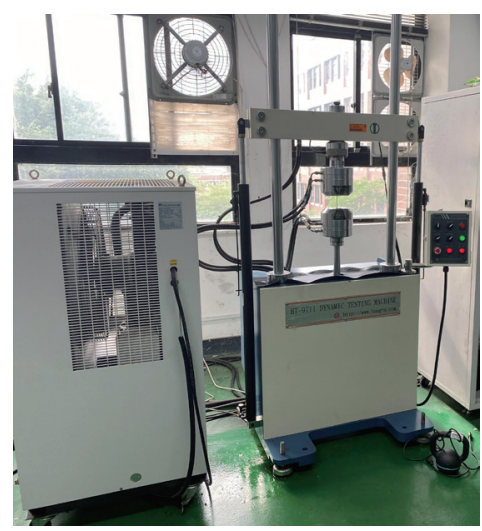

Fig. 5. (Color online) Fatigue test machine.

Table 1

Tensile test data of 3D-printed porous Ti-6Al-4V samples.

\begin{tabular}{lccccc}
\hline & $\begin{array}{c}\text { Temperature } \\
\left({ }^{\circ} \mathrm{C}\right)\end{array}$ & $\begin{array}{c}0.2 \% \text { yield } \\
(\mathrm{MPa})\end{array}$ & $\begin{array}{c}\text { Young's } \\
\text { coefficient }(\mathrm{GPa})\end{array}$ & $\begin{array}{c}\text { Tensile strength } \\
(\mathrm{MPa})\end{array}$ & $\begin{array}{c}\text { Elongation } \\
(\%)\end{array}$ \\
\hline \multirow{5}{*}{ Two rows of holes } & As-fabricated & 106.205 & 15.77 & 106.205 & 0.16 \\
& 500 & 181.28 & 23.20 & 181.28 & 1.39 \\
& 700 & 62.03 & 18.49 & 62.03 & 1.72 \\
\multirow{2}{*}{ One row of holes } & 925 & 36.22 & 16.16 & 92.63 & 1.84 \\
& 500 & 254.46 & 31.342 & 254.76 & 0.21 \\
& 700 & 181.5 & 35.75 & 183.48 & 0.15 \\
\hline
\end{tabular}

rows of holes in the lateral side was 128941 cycles (as shown in Fig. 6). The fatigue lifetime of the sample with one row of holes in the lateral side was $2 \%$ longer than that of the sample with two rows of holes. When the vibration strength was 0.3 times the yield strength, the fatigue lifetimes of the samples with one and two rows of holes in the lateral side were 144907 and 


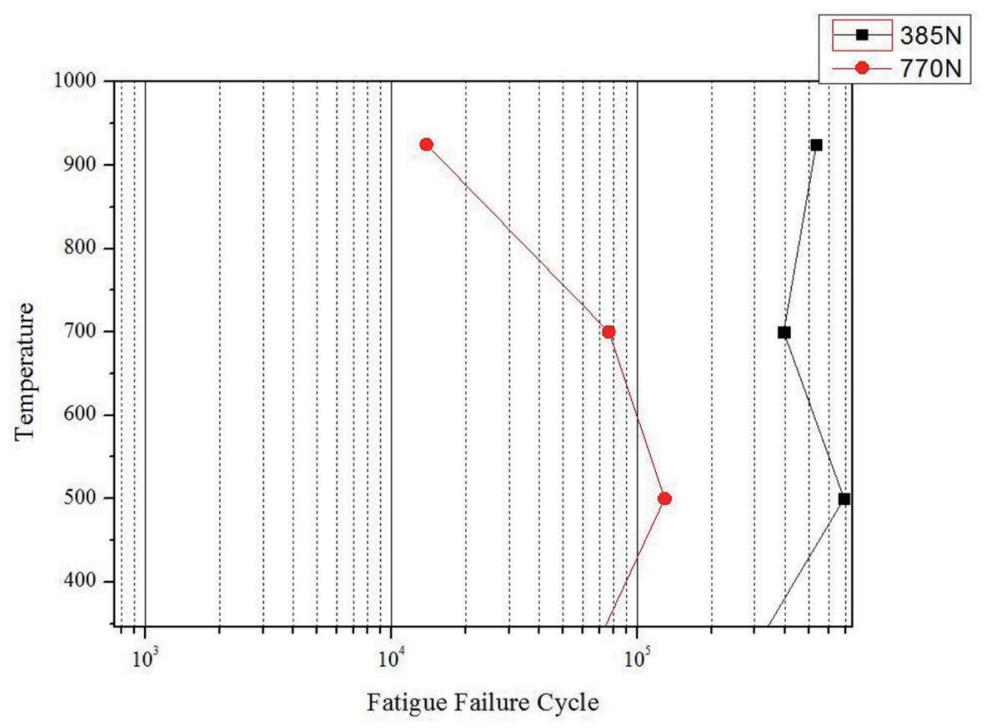

Fig. 6. (Color online) Temperature-time curves obtained from fatigue tests.

22721 times, respectively. Compared with the sample with two rows of holes in the lateral side, the sample with one row of holes had a 6.37-times longer fatigue lifetime.

\subsection{OM observation of Ti-6Al-4V samples with various heat treatments}

After performing tensile and fatigue tests, we analyzed the microstructural characteristics of the Ti-6Al-4V samples. First, we used OM to observe the samples. Figures 7(a)-7(d) show OM images of the as-fabricated, 500-, 700-, and $925-^{\circ} \mathrm{C}$-treated samples, respectively. Compared with the as-fabricated Ti-6Al-4V sample, the crystal grains of the heat-treated samples were larger. Moreover, the crystal grain size increased with the treatment temperature. A change in the microstructure of the Ti-6Al-4V sample will affect the mechanical properties, including tensile strength, elongation, and hardness. In these OM images, the bright and dark parts correspond to $\alpha$ - and $\beta$-phases, respectively. The prior $\beta$-phase with column grains along the direction of $3 \mathrm{D}$ printing was formed in the 500 - and $700{ }^{\circ} \mathrm{C}$-treated samples. In the $925-^{\circ} \mathrm{C}$-treated sample, $\alpha$-phases were changed into $\beta$-phases, causing the prior $\beta$-phase with column grains to disappear. After heat treatment at $700{ }^{\circ} \mathrm{C}$, the crystal grains were much larger than those in the $500-^{\circ} \mathrm{C}$-treated sample,even though the etching process was the same as that of other heat-treated samples. When the sample was treated at $925{ }^{\circ} \mathrm{C}$ for $1 \mathrm{~h}$, the needle-like structures became thickest [as shown in Fig. 7(d)].

\subsection{SEM observations of tensile fracture sections}

To observe and compare the morphological features of fracture sections, after the tensile tests, SEM observations were performed on the 3D-printed porous Ti-6Al-4V samples heattreated at various temperatures, as shown in Figs. 8(a)-8(d). The size of the powder particles on the fracture surface increased with increasing heat treatment temperature. According to our 

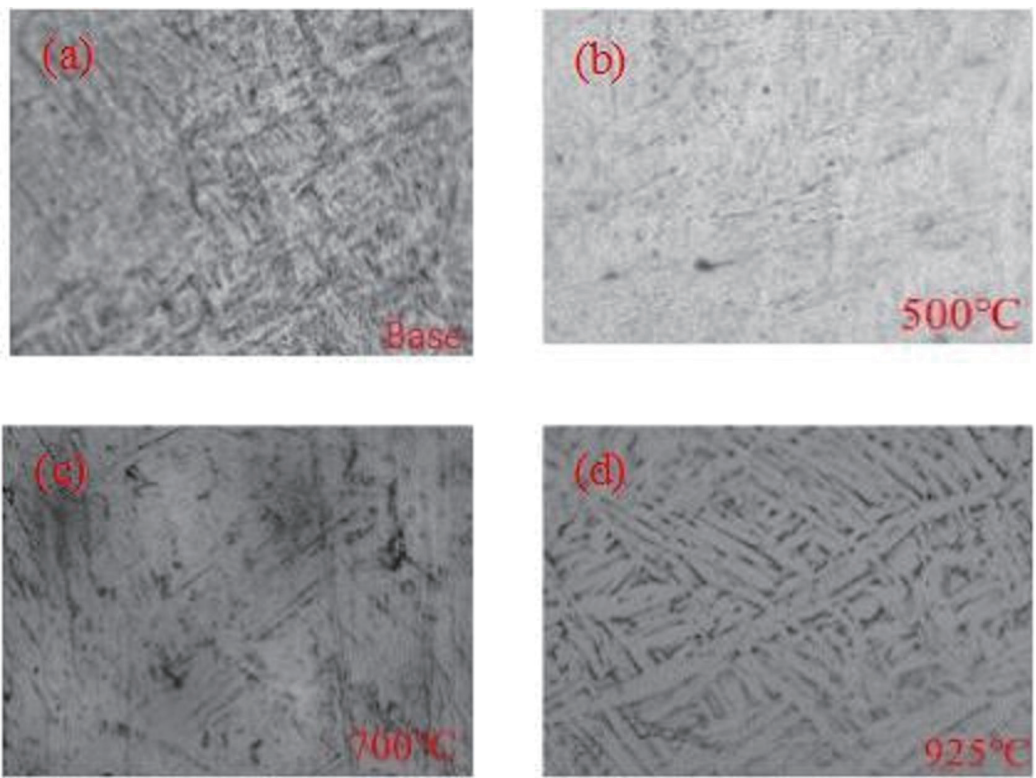

Fig. 7. (Color online) $\mathrm{OM}$ images of (a) as-fabricated, (b) 500-, (c) 700-, and (d) $925-^{\circ} \mathrm{C}$-treated $3 \mathrm{D}$-printed Ti-6Al-4V samples.
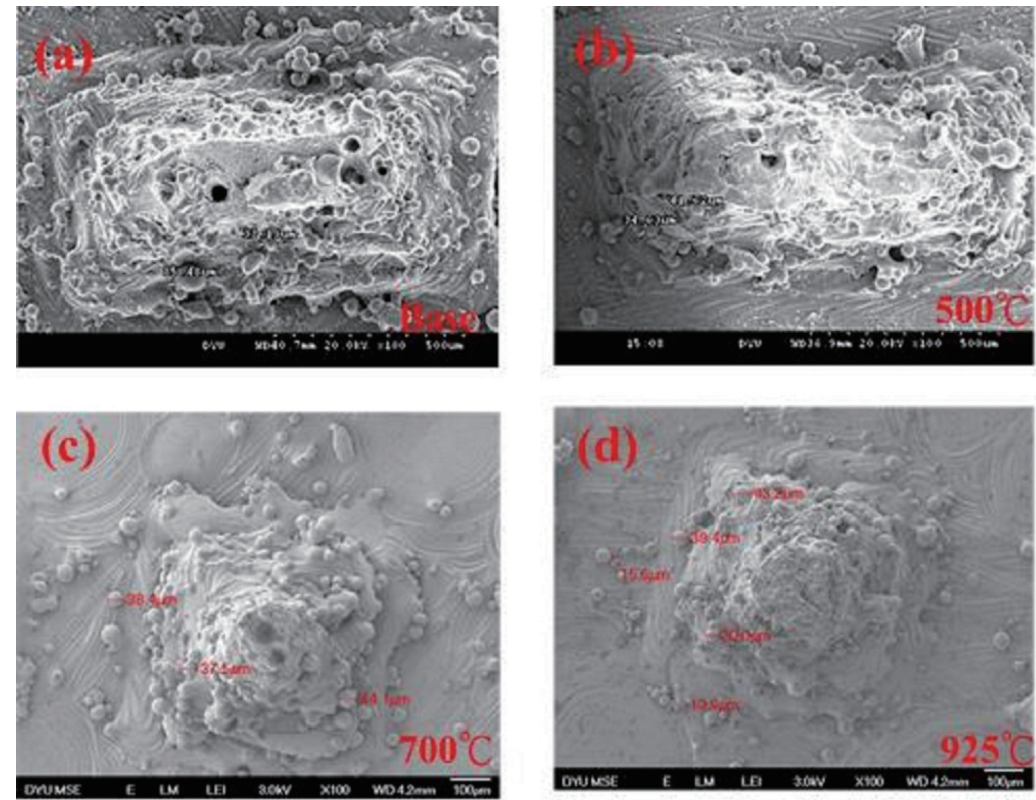

Fig. 8. (Color online) SEM images of fracture surfaces of (a) as-fabricated, (b) 500-, (c) 700-, and (d) 925- ${ }^{\circ} \mathrm{C}-$ treated 3D-printed Ti-6Al-4V samples.

analysis, the average size of the powder particles of the as-fabricated sample was $88.5 \mu \mathrm{m}$, compared with $32.3 \mu \mathrm{m}$ for the powder particles of the $925-{ }^{\circ} \mathrm{C}$-treated sample. In addition, there were fewer powder particles on the surface of the $925-^{\circ} \mathrm{C}$-treated sample than on the surface of the $500-{ }^{\circ} \mathrm{C}$-treated sample. This indicated that the fracture surface gradually became smoother with increasing heat treatment temperature. 


\section{Conclusion}

In this study, the effects of heat treatment and hole arrangement on the mechanical properties of SLM-prepared 3D-printed porous Ti-6Al-4V samples were examined. After the post-heat treatment, a structural transformation from the $\alpha$-phase to the $\beta$-phase occurred in the Ti-6Al$4 \mathrm{~V}$ sample, which affected its tensile and yield strengths. Moreover, it was found by $\mathrm{OM}$ and SEM that the heat treatments affected both the crystal structure and fracture surface morphology, respectively.

(1) The highest tensile strength was observed for the Ti-6Al-4V sample subjected to heat treatment at $500{ }^{\circ} \mathrm{C}$. This was attributed to the retention of the $\beta$-phase and the precipitation of the $\alpha$-phase, which resulted in increased ductility. However, a similar increase in the yield strength of the $500-{ }^{\circ} \mathrm{C}$-treated sample was not observed.

(2) According to the results of fatigue tests, the best mechanical properties were obtained for the 3D-printed porous Ti-6Al-4V sample with four rows of holes in the front side and one row of holes in the lateral side after performing heat treatment at $500{ }^{\circ} \mathrm{C}$ for $4 \mathrm{~h}$.

\section{References}

1 Q. S. Wang, S. J. Li, W. T. Hou, R. Yang, and R. D. K. Misra: J. Mech. Behav. Biomed. Mater. 103 (2020) 103590. https://www.sciencedirect.com/science/article/pii/S0144861716304039

2 B. Baufeld, E. Brandl, and O. Biest: J. Mater. Process. Technol. 211 (2011) 1146. https://www.sciencedirect. com/science/article/pii/S0924013611000306

3 S. Q.Wu, Y. J. Lu, T. T. Huang, C. Q. Zhao, J. J. Lin, S. Guo, and J. X. Lin: J. Alloys Compd. 672 (2016) 643. https://www.sciencedirect.com/science/article/pii/S0925838816304510\#!

4 R. Molaei, A. Fatemi, N. Sanaei, J. Pegues, S. Shao, P. Li, D. H. Warner, and N. Phan: Int. J. Fatigue 132 (2020) 10563. https://www.sciencedirect.com/science/article/pii/S0142112319304670\#!

5 B. Vrancken, L. Thijs, J. P. Kruth, and J. V. Humbeeck: J. Alloys Compd. 541 (2012) 177. https://www. sciencedirect.com/science/article/pii/S0925838812011826\#!

6 H. Yu, F. Li, and X. Zeng: Int. J. Fatigue 120 (2019) 175. https://www.sciencedirect.com/science/article/pii/ S014211231830714X\#!

7 S. Leuders, M. Thöne, A. Riemer, T. Niendorf, T. Tröster, H. A. Richard, and H. J. Maier: Int. J. Fatigue 400 (2013) 300. https://www.sciencedirect.com/science/article/pii/S014211231200343X\#!

8 C. N. Kelly, N. T. Evans, C. W. Irvin, S. C. Chapman, K. Gall, and D. L. Safranski: Mater. Sci. Eng. C. 198 (2019) 726. https://www.sciencedirect.com/science/article/pii/S0928493118321350\#!

9 X. Jin, L. Lan, S. Gao, B. He, and Y. Rong: Mater. Sci. Eng., A 780 (2020) 139199. https://www.sciencedirect. com/science/article/pii/S0921509320302859

\section{About the Authors}

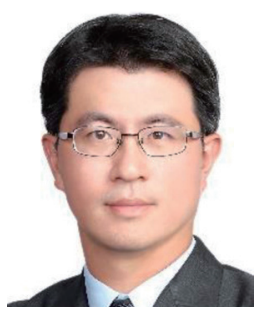

Ming-Hsien Hu received his B.S. degree from Taipei Medical University, Taiwan, in 2004 and his Ph.D. degree from National Cheng Kung University, Taiwan, in 2018. Since 2020, he has been the director of the orthopedic department of Show Chwan Memorial Hospital, Taiwan. His research interests are in spine surgery, biomedical devices, and medical engineering. (minghsienhu@gmail.com) 


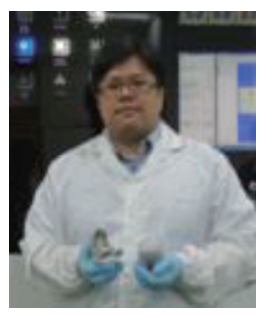

Chun-Ming Chang received his B.S., M.S., and Ph.D. degrees from Southern Taiwan University of Science and Technology, National Taiwan Normal University, and National Taiwan University, Taiwan, in 2001, 2006, and 2014, respectively. Since 2017, he has been an associate researcher at the Instrument Technology Research Center, National Applied Research Laboratories, Taiwan. His research interests are in 3D printing, implant material research, and material science. (gmp@itrc.narl.org.tw)

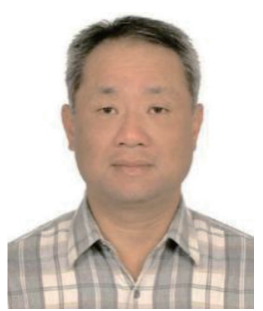

Tan-Chih Chang received his B.S. degree from Feng Chia University, Taiwan, in 1986 and his M.S. degree from Feng Chia University, Department of Civil Engineering, Taiwan, in 1989. He is currently a Ph.D. student. Since 2018, he has been a researcher at Da-Yeh University, Taiwan. His research interests include 3D printing, the manufacture of composite materials, tensile testing in carbon fiber research, voice control, and computer graphics. (hiciv@ms43.hinet.net)

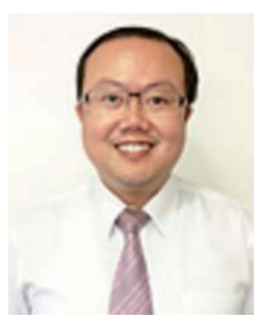

Yao-Tsung Yang received his M.S. and Ph.D. degrees from Da-Yeh University, Taiwan, in 1993 and 1997, respectively. Since 2017, he has been an associate professor at Da-Yeh University, Taiwan. His main areas of interest include dental implantology, prosthodontology, 3D printing, the manufacture of composite materials, tensile test research, control, and computer graphics. (p2410@mail.dyu.edu.tw)

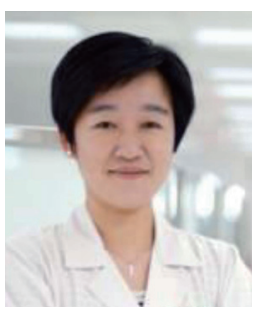

Chia-Hui Chien received her DDS from Kaohsiung Medical University, Taiwan, in 2004. She is currently an attending staff member in Chi-Mei Medical Center and an assistant professor in Chung Shan Medical University. She was a visiting scholar in the University of North Carolina for one year in 2018. Her main areas of interest include dental implantology, prosthodontology, and esthetic dentistry. (t0124982@hotmail.com)

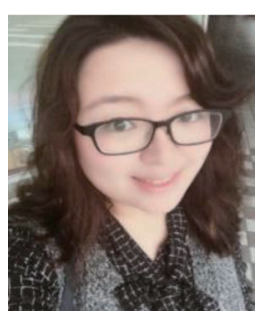

Wan Ao received her B.S. degree from Da-Yeh University, Taiwan, in 2018. From 2018 to 2019, she was a researcher at Da-Yeh University. Her research interests are in 3D metal printing, testing, implant material development, and computer graphics. (R0790015@ mail.dyu.edu.tw)

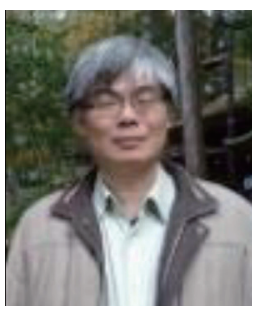

Feng-Min Lai received his B.S. degree from Chinese Culture University, Taiwan, in 1991 and his M.S. and Ph.D. degrees from National Chiao Tung University, Taiwan, in 1993 and 1997, respectively. From 2002 to 2016, he was an associate professor at Da-Yeh University, Taiwan, where he has been a professor since 2017. His research interests are in composite materials, computer graphics, and medical aid development. (fengmin@mail.dyu.edu.tw) 